\title{
Probing the magnetic topology of coronal mass ejections by means of Ulysses/HI-SCALE energetic particle observations
}

\author{
O. Malandraki, E. T. Sarris, P. Trochoutsos \\ Demokritos University of Thrace, Department of Electrical Engineering, Xanthi, 67100, Greece
}

Received: 21 April 1999 / Revised: 23 August 1999 / Accepted: 30 August 1999

\begin{abstract}
In this work, solar flare energetic particle fluxes $\left(\mathrm{E}_{\mathrm{e}} \geq 42 \mathrm{keV}\right)$ observed by the HI-SCALE instrument onboard Ulysses, a spacecraft that is probing the heliosphere in 3-D, are utilized as diagnostics of the large-scale structure and topology of the interplanetary magnetic field (IMF) embedded within two well-identified interplanetary coronal mass ejection (ICME) structures. On the basis of the energetic solar flare particle observations firm conclusions are drawn on whether the detected ICMEs have been detached from the solar corona or are still magnetically anchored to it when they arrive at 2.5 AU. From the development of the angular distributions of the particle intensities, we have inferred that portions of the ICMEs studied consisted of both open and closed magnetic field lines. Both ICMEs present a filamentary structure comprising magnetic filaments with distinct electron anisotropy characteristics. Subsequently, we studied the evolution of the anisotropies of the energetic electrons along the magnetic field loop-like structure of one ICME and computed the characteristic decay time of the anisotropy which is a measure of the amount of scattering that the trapped electron population underwent after injection at the Sun.
\end{abstract}

Key words: Interplanetary physics (energetic particles; interplanetary magnetic fields)

\section{Introduction}

The presence of huge magnetic loops that could retain their connection to the Sun and extend to distances beyond the Earth's orbit has been suggested in the past (Gold, 1959; Cocconi et al., 1958). Moreover, it has

Correspondence to: E. T. Sarris been hypothesized that magnetic loops could even be detached from the Sun to form 'magnetic bubbles' (Piddington, 1958). The white light coronagraph experiment aboard Skylab directly observed mass ejections from the Sun, which propagated as magnetic bubbles through the corona while outer ends obviously remained attached to the Sun (Gosling et al., 1974).

Rao et al. (1967) made the first attempt to probe the topology of interplanetary fields using solar energetic particles (SEPs). In their survey of energetic storm particle events observed by Pioneer 6 and 7, they found four periods of bi-directional anisotropies of $10 \mathrm{MeV}$ protons, all occurring during the maximum depressions of Forbush decreases. They argued that their observations were not consistent with a Gold bottle (i.e. attached magnetic bottle geometry) but were better explained by particles behind a blast wave in an open field line configuration. Palmer et al. (1978) found 16 periods of bi-directional streaming of low energy solar particles with an average duration of $9 \mathrm{~h}$ behind shock waves. Contrary to Rao et al. (1967) they argued in favour of a Gold bottle. Kutchko et al. (1982) analyzed an SEP event on October 12, 1977, that exhibited $1 \mathrm{MeV}$ proton and alpha particle field-aligned bi-directional fluxes for about $5 \mathrm{~h}$. Electron anisotropies were also bi-directional but were peaked perpendicular to the magnetic field. The Gold bottle was compared with a pinched-off bottle, or plasmoid and the former configuration was considered to be the most consistent. Sarris and Krimigis (1982) have presented IMP-7 observations of energetic particles injected by solar flares into extended solar magnetic loop-like structures. From the development of the angular distributions of the intensities of energetic protons $\left(E_{p} \geq 300 \mathrm{keV}, E_{p} \geq 25 \mathrm{MeV}\right)$ and electrons $\left(E_{e} \geq 220 \mathrm{keV}\right)$ they have inferred that energetic particles are bouncing between two magnetic mirrors located at the Sun and have obtained for the first time estimates of the extent of magnetic loops to distances 3.5 AU from the Sun. Marsden et al. (1987) have presented the results of a survey of low-energy ion bidirectional anisotropies and associated transient mag- 
netic structures as observed in the energy range $35-1600 \mathrm{keV}$ on board ISEE-3 at the time of peak activity of sunspot cycle 21 . From a comparison of the anisotropy signatures at 35 and $620 \mathrm{keV}$ they concluded that their observations of the CME-related magnetic structures were most consistent with detached bubbles comprising closed loops or tightly wound cylindrical helices rather than extended tonguelike loops attached to the Sun at the time of observation.

Kahler and Reames (1991) showed evidence of a flare particle onset in one magnetic cloud, suggesting that the magnetic field lines were still attached to the Sun when the magnetic cloud arrived at $1 \mathrm{AU}$. Farrugia et al. (1993) have detected streaming particle beams of coronal origin inside a magnetic cloud at $1 \mathrm{AU}$ and suggested that magnetic field lines embedded within the magnetic cloud remained connected to the Sun. Armstrong et al. (1994) observed hot $(\sim 270 \mathrm{keV})$ coronal particles at $4.6 \mathrm{AU}$ and $32^{\circ}$ south heliolatitude, during the passage of a CME which had an internal structure of a large magnetic flux rope. They concluded that the beams observed by HI-SCALE can be explained if the flux rope were rooted near a coronal energetic particle source thus providing a 'conduit' for these particles along its axis. Pick et al. (1995) have identified at $3.5 \mathrm{AU}$ and $54^{\circ}$ south heliolatitude a particle propagation channel inside an ICME which was filled by solar flare particles. Bothmer et al. (1995) have found a class of SEP events from observations of the Ulysses spacecraft at high heliospheric latitudes where energetic particles have been convected outward from the Sun within CMEs.

Gosling et al. (1995) suggested that sustained 3dimensional magnetic reconnection, occurring within the rising loops of a CME, can produce a mixture of magnetic topologies, namely open field lines connected to the Sun at only one end, looped magnetic field lines connected at both ends to the solar corona and field lines that are connected to the outer heliosphere at both ends. Bothmer et al. (1996) observed bi-directional and streaming anisotropy suprathermal electron and 0.4$5 \mathrm{MeV}$ ion flux characteristics within two different portions of an ICME detected by Ulysses, which they interpreted as evidence of closed magnetic loops and open magnetic field lines, respectively. Recently, Richardson (1997) has reviewed several ways in which energetic particle observations provide information on the structure of ejecta (the interplanetary manifestations of solar mass ejections). He states that field-aligned bi-directional energetic $(\sim \mathrm{MeV})$ ion flows, a feature observed within many ejecta, are generally interpreted in terms of particles circulating within a plasmoid region disconnected from the Sun or being reflected within the legs of looped magnetic field lines rooted at the Sun. Fischer et al. (1997), using IMP-8 high time resolution observations of protons $(0.3-0.5 \mathrm{MeV})$ and electrons $(>16 \mathrm{keV}$ ), have found within one magnetic cloud and its environs successive intervals of distinct bidirectional proton fluxes and of nearly isotropic fluxes. They interpret this particle behaviour as a result of the complicated internal structure of clouds or their vicinity that may have the character of deformed layers or tubes, with some magnetic field lines connected to and others disconnected from the Sun. Furthermore, Larson et al. (1997) have presented WIND observations of five impulsive $\sim 1-10^{2} \mathrm{keV}$ solar flare electron events inside the magnetic cloud observed upstream of the Earth on October 18-20, 1995. Although the cloud magnetic field exhibited the smooth, continuous rotation signature of a helical flux rope, the $\sim 0.1-1 \mathrm{keV}$ heat flux electrons and $\sim 1-10^{2} \mathrm{keV}$ energetic electrons showed numerous simultaneous abrupt changes from bidirectional streaming to uni-directional streaming to complete flux dropouts. They have interpreted these observations as evidence that the cloud comprised magnetic field lines connected to the Sun on both ends, on one end, and completely disconnected, intertwined together. Malandraki et al. (2000) have presented Ulysses observations of a solar flare electron event onset inside a well-identified ICME. The impulsive profile of the event indicated the particles had direct access to Ulysses after their injection at the Sun. The solar energetic electrons were found to be associated with a solar type III burst activity and were streaming in a collimated beam along magnetic field lines embedded within the ICME. They concluded that the SEP observations strongly suggest that the field lines embedded in the ICME structure remained connected to the Sun after the CME lift-off in the solar corona.

In this work, we study the large-scale topology of the IMF embedded within two well-identified ICME structures, based upon energetic electron observations by the ULYSSES/HI-SCALE instrument. The question of whether the detected ICMEs have been detached from the solar corona or are still magnetically anchored to it when they arrive at $2.5 \mathrm{AU}$ is addressed.

\section{Instrumentation}

In this work, hourly averaged measurements of the angular distribution of the intensities of energetic electrons in the energy range $42-290 \mathrm{keV}$, detected by the sunward looking telescope LEFS60 (Low-Energy Foil Spectrometer) of the HI-SCALE experiment (Heliosphere Instrument for Spectra, Composition and Anisotropy at Low Energies) onboard ULYSSES, are utilized. The LEFS60 telescope has a geometrical factor of $\sim 0.48 \mathrm{~cm}^{2} \mathrm{sr}$. The number 60 denotes the angle that the collimator centerline of the telescope makes with the Ulysses spin axis. The HI-SCALE experiment has been described in full detail by Lanzerotti et al. (1992). In the sunward looking LEMS30 ion telescope (Low-Energy Magnetic Spectrometer), mounted at $30^{\circ}$ with respect to the spacecraft axis, electrons with energies below $\sim 300 \mathrm{keV}$ are swept away from the ion detectors of the telescope and counted by the separate detector B, of the composition aperture (CA; sometimes referred to in the Ulysses project as the 'WART'). Hourly and spin-averaged measurements of these magnetically deflected electrons (DE) in the energy ranges DE1 (38$53 \mathrm{keV})$, DE2 (53-103 keV), DE3 (103-175 keV) and 
DE4 (175-315 keV) are also reported. The WART B detector head has a geometrical factor of $\sim 0.05 \mathrm{~cm}^{2} \mathrm{sr}$.

The Ulysses magnetic field experiment consists of a fluxgate magnetometer (FGM) and a vector helium magnetometer (VHM) which measure the three orthogonal components of a magnetic field vector (Balogh et al., 1992). Hourly averaged IMF measurements are used.

\section{Observations}

In Fig. 1, an overview of the hourly and spin-averaged counting rates of four electron channels $(42-290 \mathrm{keV})$ of the HI-SCALE experiment is presented for the interval from 1200 UT, March 23, 1991 to March 29, 1991 (1200 UT on day 82 to day 88) (top panel). Shaded bars in the top panel show intervals that correspond to two ICME structures as identified by Phillips et al. (1992) and Phillips (1997). Counterstreaming halo electron beams, which are considered to be the most reliable signature of the passage of an ICME (e.g. Gosling, 1990), have been observed throughout the two regions. The first ICME period, bounded in Fig. 1 by vertical traces $\mathrm{C} 1$ (commencement 1) and E1 (end 1), began at 0631 UT on March 24 and ended at 1140 UT on March 26. This ICME had the characteristic plasma and field signatures of a magnetic cloud. It has been associated with a fast forward shock observed at 1543 UT on March 23 (Balogh et al., 1995), marked by the vertical dashed line $\mathrm{S} 1$ in Fig. 1. Furthermore, this interval includes a shock observed at 0552 on March 25, marked by a vertical dashed trace labeled S2 (Balogh et al., 1995). The second ICME interval, marked by vertical traces C2 and E2 in Fig. 1, started at 0915 UT on March 27 and ended at 1200 UT on March 29. It was associated with the S2 fast forward shock. This shock has a $\theta_{\mathrm{Bn}}$ angle equal to $47^{\circ} \pm 17^{\circ}$ (Balogh et al., 1995). By definition, $\theta_{\mathrm{Bn}}$ is the acute angle between the normal to the shock surface and the upstream magnetic field. Thus, this shock is classified as an oblique shock. No active in situ shock acceleration is evident since there is no shockassociated enhancement.

As it can be seen in Fig. 1, the sequence of the two ICMEs is observed around the peak and during the decay phase of a major pure solar flare electron event (see Discussion). Comparison of the intensity profile of the LEFS60 foil spectrometer (top panel) with the intensity profile of the magnetically-deflected electrons measured by the WART B detector (second panel) reveals that the former follows closely the latter in structure during this period. Thus, it is concluded that the LEFS60 response is primarily due to electrons. As the geometrical factor of the LEFS60 telescope is larger than that of the WART B detector head, electron measurements from the LEFS60 telescope are used in this work. The three lower panels in Fig. 1 show 1-h averaged IMF magnitude and direction in the heliocentric RTN system for the same period. The RTN coordinate system uses the Sun-Ulysses direction $(\mathrm{R})$, prograde heliocentric orbital direction $(\mathrm{T})$, and the right hand completion $(\mathrm{N})$.
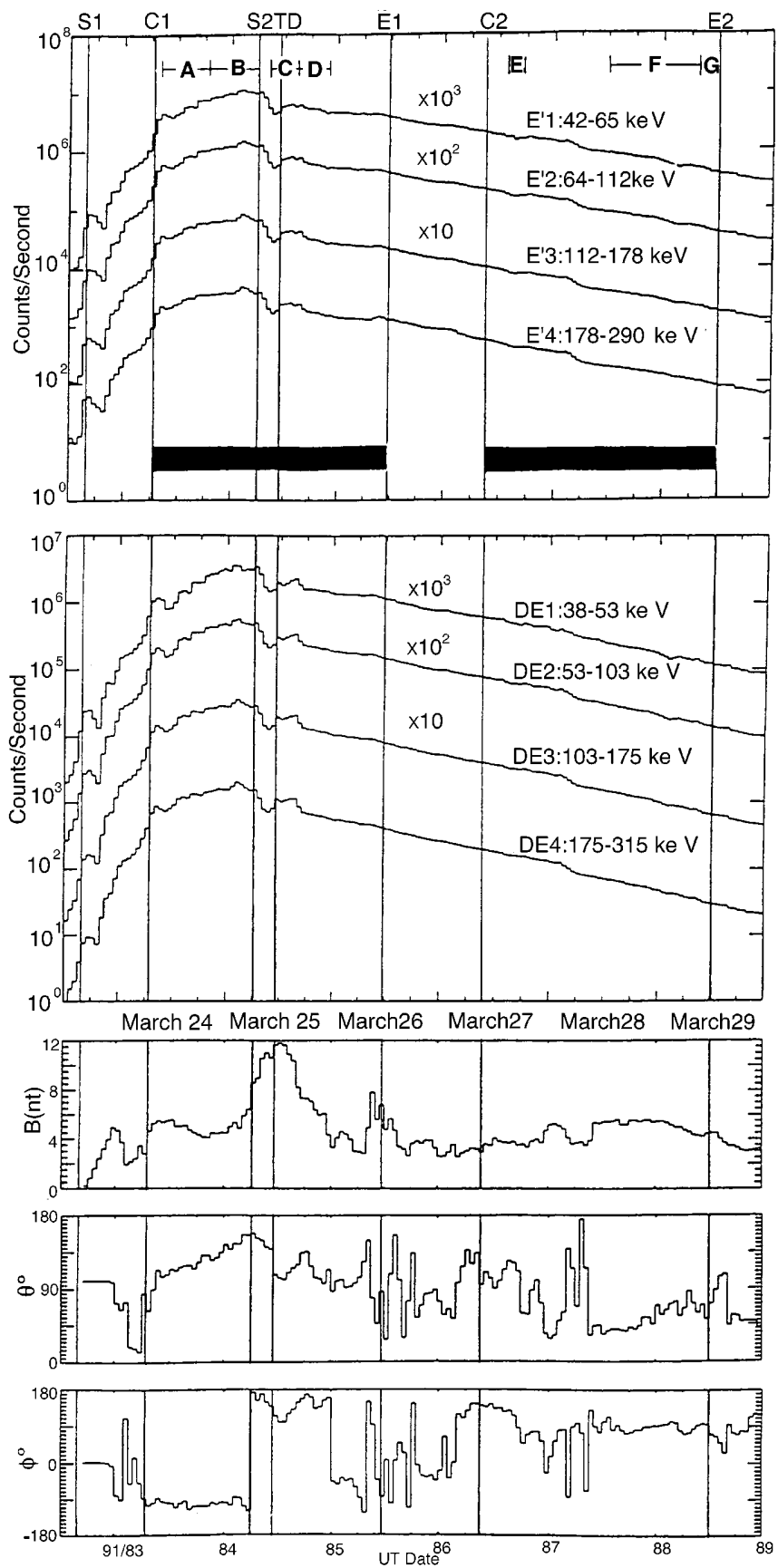

Fig. 1. Ulysses energetic electron and magnetic field data from 23 12:00 UT-29 March 1991 (days 82 12:00 UT - 88). Top to bottom: $42-$ $290 \mathrm{keV}$ hourly and spin-averaged electron counting rates, as measured by the sunward looking LEFS60 telescope, $38-315 \mathrm{keV}$ hourly and spin-averaged counting rates of magnetically deflected electrons measured by the WART B detector, IMF magnitude, latitude $\theta$, and azimuth angle $\phi$ of the magnetic field vector, as measured by the VHM/FGM magnetometer in RTN local heliospheric coordinates. Shaded bars show the two ICME intervals. Vertical traces $S 1, S 2, C 1, C 2, E 1, E 2$ and TD mark the two shocks, the commencement and end of the two ICMEs and the tangential discontinuity observed within the first ICME. Also depicted are the extended regimes (A-G) observed during the passage of the ICMEs over Ulysses that exhibited distinctly different and unusual electron anisotropy characteristics 
A noteworthy feature observed within the first ICME is a discontinuity visible in the deflection of the polar angle on March 25 (day 84 at 1100 UT, Fig. 1). Using the interplanetary magnetic field data, calculations show that the magnetic field vectors $\mathbf{B}_{\mathbf{1}}$ and $\mathbf{B}_{\mathbf{2}}$ on opposite sides of the discontinuity satisfy the following conditions:

$\operatorname{angle}\left(\mathbf{B}_{1}, \mathbf{B}_{2}\right)>\arccos \left(B_{1} / B_{2}\right)$

$\left|\left(\mathbf{B}_{1}-\mathbf{B}_{2}\right) / B_{\max }\right| \leq 0.4$

which indicates that the discontinuity is most probably tangential (Burlaga, 1968). Tangential discontinuities act as barriers in interplanetary space, separating different particle populations and IMF regimes (Maia et al., 1998). On this day, the tangential discontinuity (TD) crossed by Ulysses is obviously separating the regime with the smooth decay phase of the particle intensities from the upstream modulated electron intensities.

Careful inspection of the pitch angle distributions (PADs) of the solar electron population observed during the passage of the ICMEs over Ulysses, reveals the existence of regions exhibiting different electron aniso- tropy characteristics, labeled $A-G$ in Fig. 1. The time evolution of the angular distributions of the energetic electron intensities observed by Ulysses within the two ICMEs is shown in Fig. 2. Representative PAD snapshots for each region encountered by the spacecraft are presented. As depicted in Fig. 2a, electron PADs switch from weak unidirectional and isotropic outside the ICME to strongly bi-directional after Ulysses crosses the front boundary of the first ICME. Initially, after entering within the first ICME, Ulysses detects a region (labeled A in Fig. 1) where energetic electron intensities exhibit strong bi-directional anisotropies, nearly symmetrical around $90^{\circ}$ pitch angle. These bi-directional anisotropies are signatures of strong trapping of the observed electron population. Throughout this regime electron count rates have nearly reached maximum values. The spacecraft remained in this region for $\sim 11 \mathrm{~h}$. Contiguous to region $\mathrm{A}$, a region with less strong but distinct electron bi-directionality aligned along the magnetic field was encountered (region B). The flux profile during this period shows a plateau-like structure at maximum flux. The electron bi-directional distribution persisted from 1900 UT on the March 24 till the time of arrival of the shock at 0552 UT on March 25 as

\section{OUTSIDE CME}
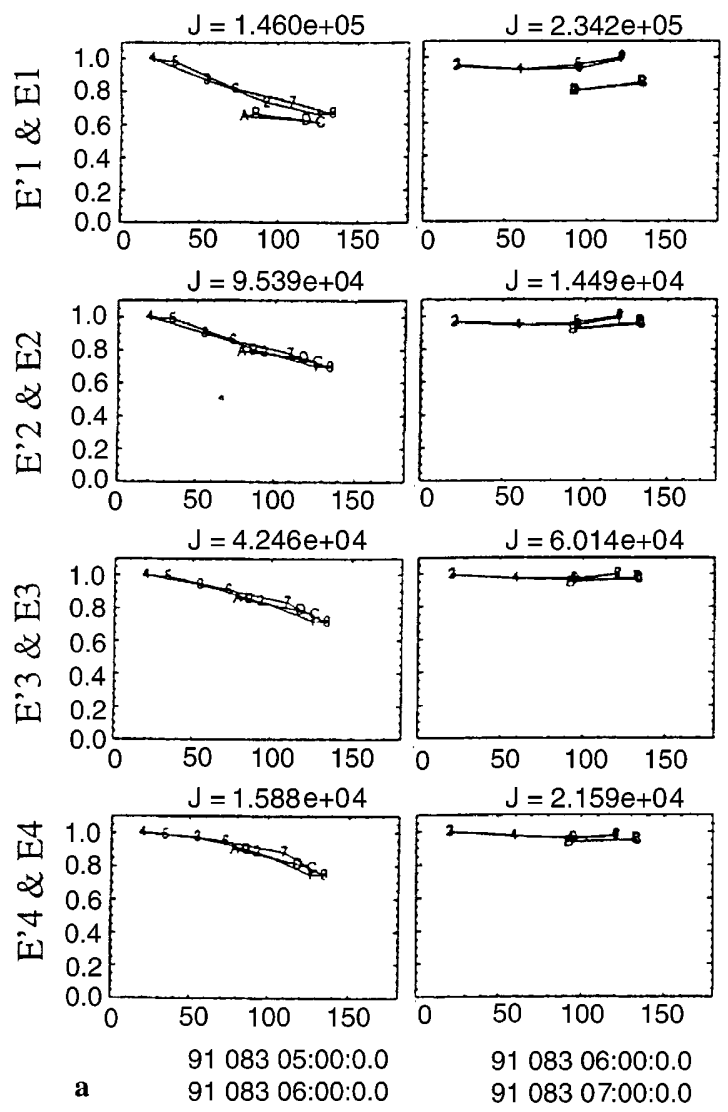

Fig. 2 a. The first two columns in this figure present electron pitch angle distributions detected before Ulysses entered the first ICME. The three subsequent columns are representative of pitch angle distribution snapshots for the trapped electron population observed in region $A$. Evidently, electron pitch angle distribution characteristics

\section{REGION “A”}

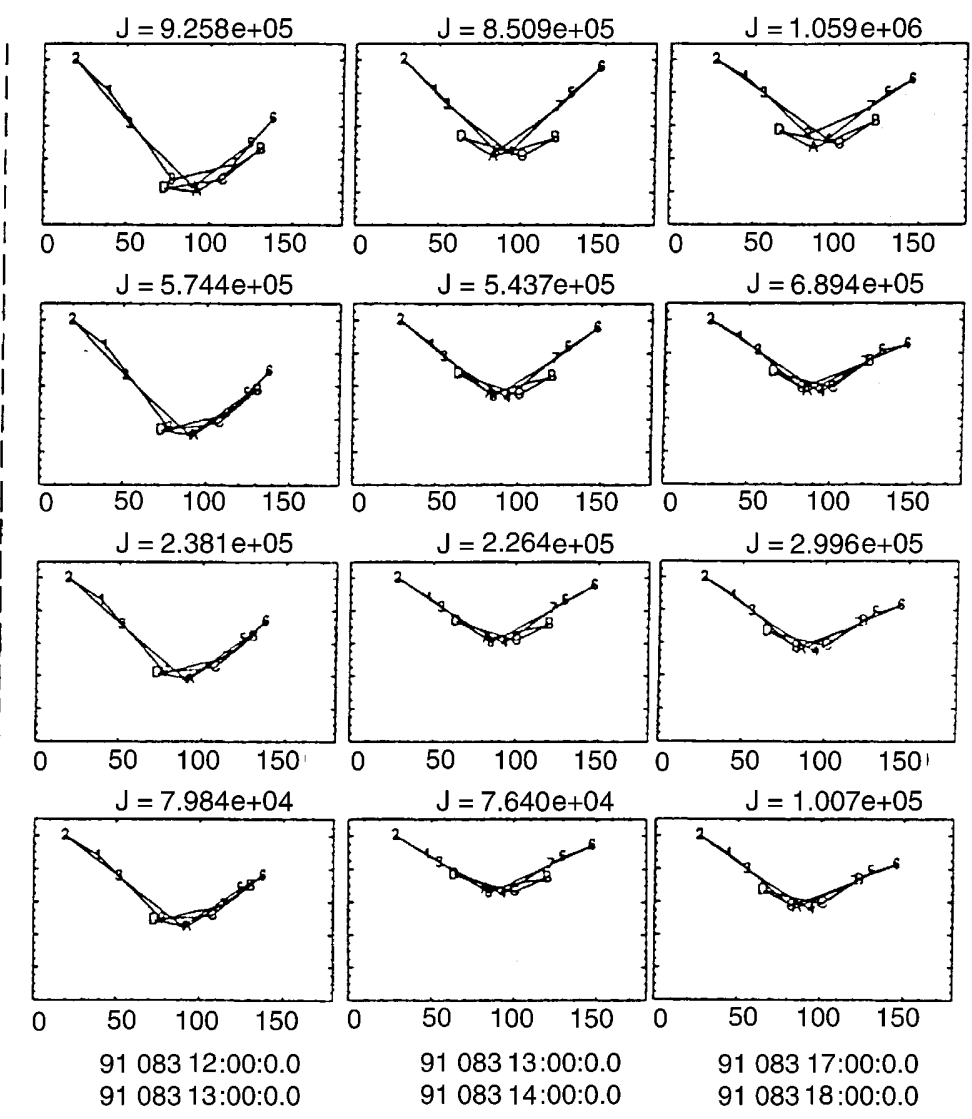

differ dramatically outside and within the ICME. Normalized differential flux is plotted versus pitch angle. Estimated instrumental backgrounds have been subtracted. The time interval is indicated below each graph. (E'1: 42-65 keV, E'2: 65-112 keV, E'3: 112$178 \mathrm{keV}, \mathrm{E}^{\prime} 4:$ 178-290 keV). 
shown in Fig. 2b. As expected, completely different electron pitch angle distributions are observed as Ulysses proceeds from the upstream into the downstream region of the shock, indicating the particle anisotropies are modulated by the shock in its immediate vicinity.

Then, it is evident that the spacecraft proceeds from a regime where strong, field-aligned bi-directional fluxes, that persist for $\sim 6 \mathrm{~h}$, are detected (region C, Fig. 2c), into a region where low unidirectional electron anisotropies lasting for $\sim 7 \mathrm{~h}$ are observed (region D, Fig. 2d). The electron anisotropy is directed anti-parallel to the IMF direction, which indicates the electron population detected in region $\mathrm{D}$ is streaming away from the Sun. Isotropic particle distributions were observed till the rear boundary of the first ICME.

Unusual particle anisotropies were also observed during the passage of the second ICME. Counterstreaming electron fluxes along the local IMF were detected within region $\mathrm{E}$ for $\sim 3 \mathrm{~h}$ (Fig. 2e). After a period with isotropic particle characteristics, Ulysses enters the extended region $F$ populated with electrons that exhibit strong field-aligned bi-directional anisotropies persistent for $\sim 20 \mathrm{~h}$ (Fig. 2f). Adjacent to region F, a regime is detected where electron unidirectional fluxes persist for $\sim 3 \mathrm{~h}$, till Ulysses exits the ICME (region $\mathrm{G}$, Fig. 2g).

\section{Discussion}

Roelof et al. (1992), using HI-SCALE observations, provided evidence that the great pulse of solar activity in February-April, 1991, created a 'reservoir' of lowenergy ions and electrons in the inner heliosphere. They concluded that the reservoir was filled up by the accumulation of the output of many large flares rather than by an isolated injection from any single great flare. Specifically, they have shown that the inner heliosphere appeared to fill up gradually (not impulsively) with electrons during the rise phase of the electron event

\section{REGION "B"}

UPSTREAM

\section{SHOCK “S2” DOWNSTREAM}
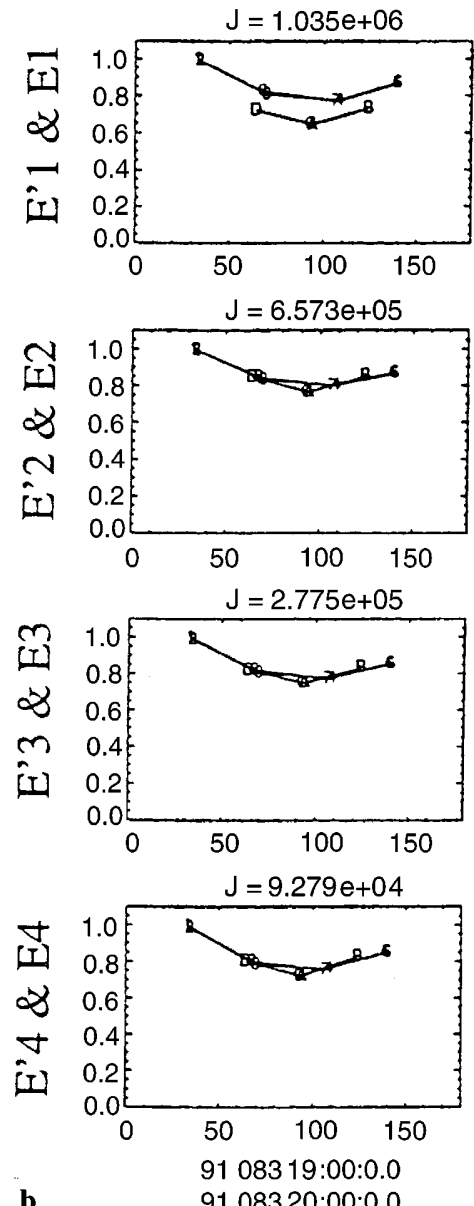
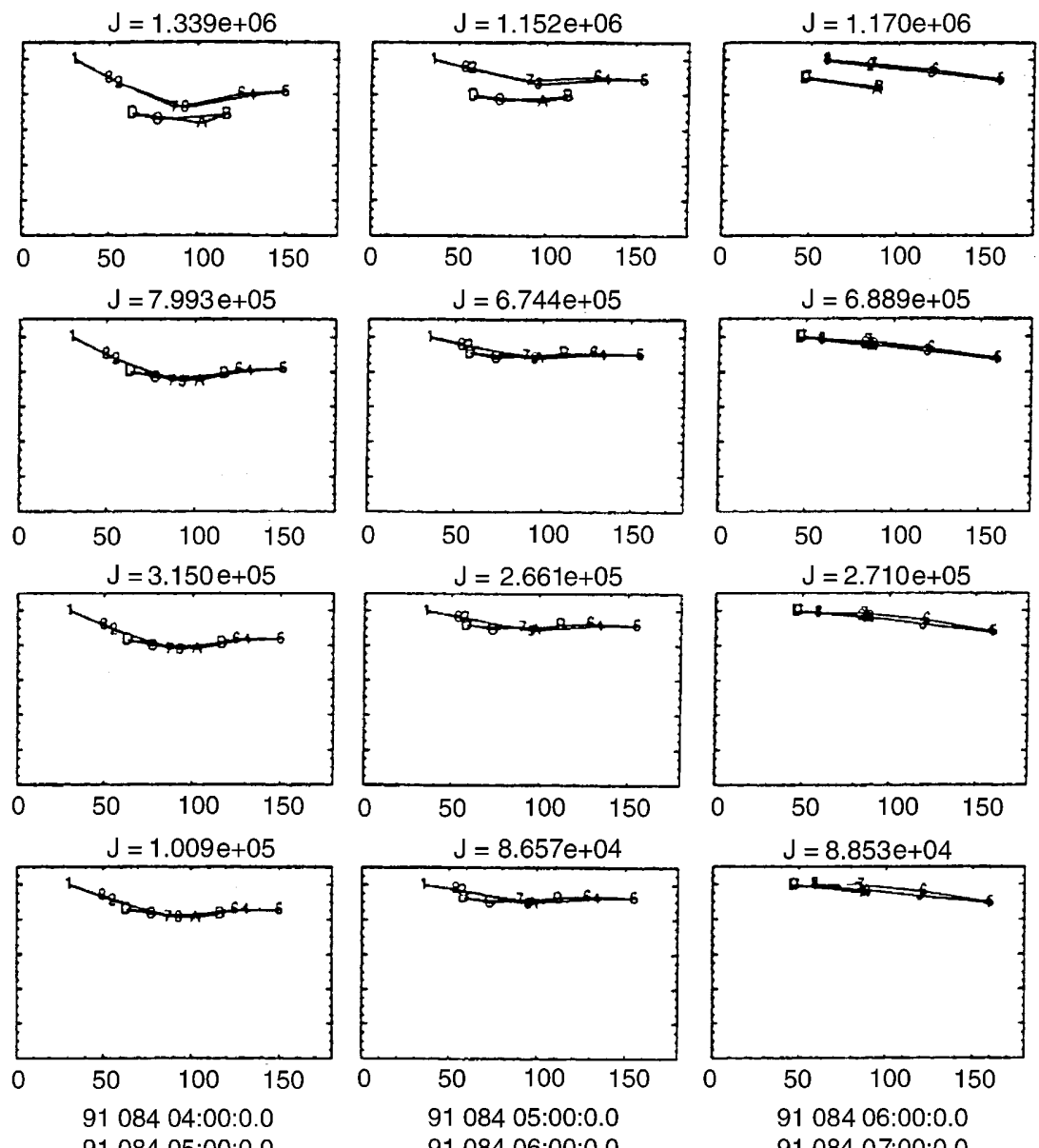

Fig. 2b. PAD snapshot for the electrons observed within region $B$, where Ulysses observes distinct electron bi-directional anisotropies. Electron anisotropy characteristics for the upstream and downstream regions of the shock, along with the PADs observed on shock arrival are also presented. Note that completely different anisotropies are observed as Ulysses proceeds from the upstream into the downstream region of the shock. 


\section{REGION “C”}
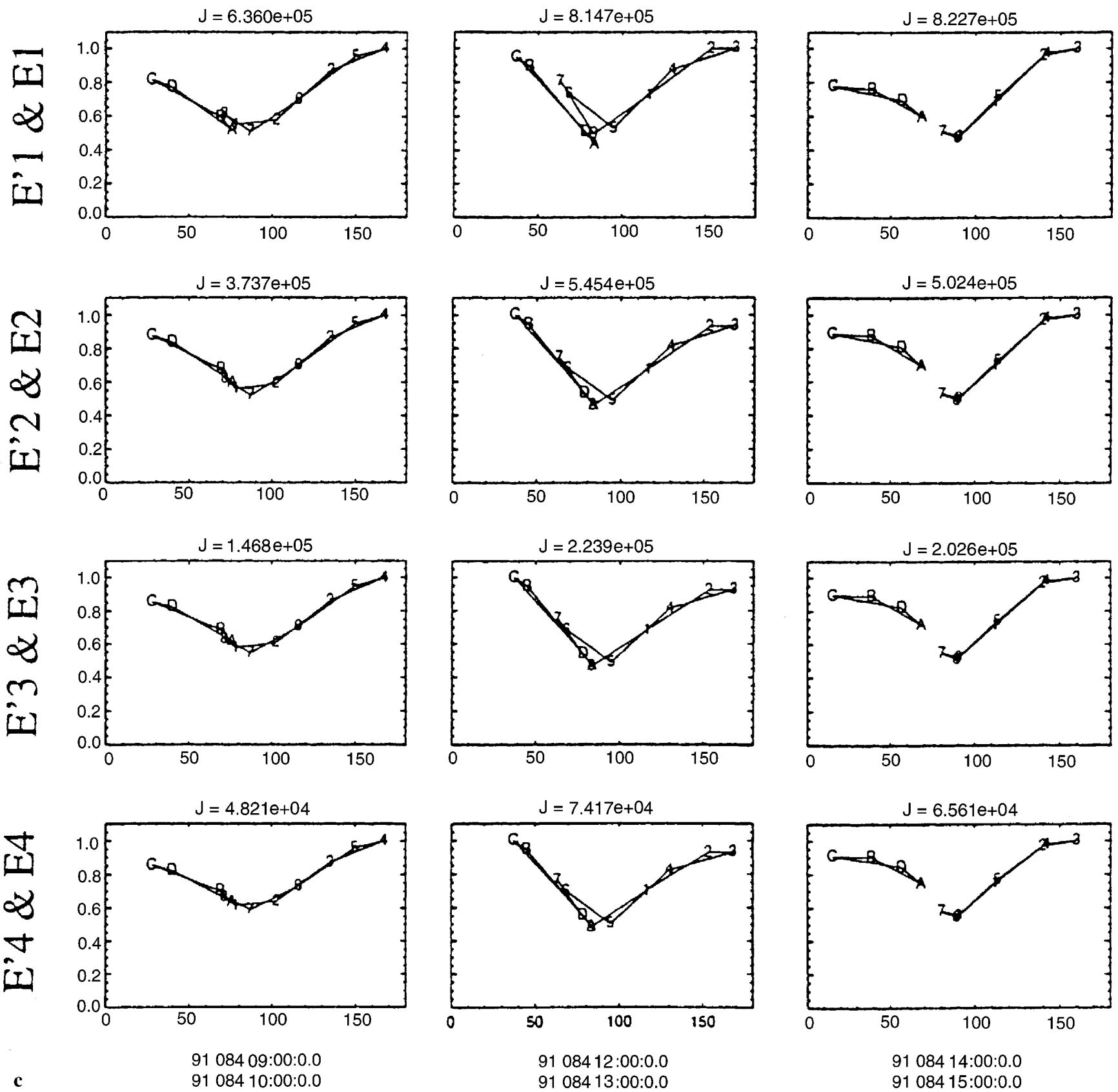

Fig. 2c. Strong field-aligned bi-directional electron fluxes are observed in region $C$, which is indicative of a trapped population in the downstream region of the shock.

shown in Fig. 1. They have associated this electron event with a great X9.4 (white light, $\mathrm{H}$-alpha importance 3B) solar flare with maximum brightness at 2245 UT on March 22 (day 81) which was located at E30 and was produced by active region 6555 . The electron fluxes started to rise nearly monotonically at 1200 UT on March 23 (day 82). The transit time for a $63 \mathrm{keV}$ electron with zero-degree pitch-angle along an ideal spiral to 2.5 AU (i.e., a curvilinear distance of $3.3 \mathrm{AU}$ for a solar wind velocity of $650 \mathrm{~km} / \mathrm{s}$ ) is one hour. Roelof et al. (1992) state that the reason the rise is nearly velocity dispersionless with a near-constant spectrum is that the transit times for these particles are considerably shorter than the rise time of $3.1 \mathrm{~h}$. Thus, the electrons easily populate the flux tube to $2.5 \mathrm{AU}$ faster than their rate of injection. On March 23, Ulysses was located at a distance of $2.5 \mathrm{AU}$ from the Sun, and the Ulysses footpoint mapped back to a longitude of 


\section{REGION" "D"}
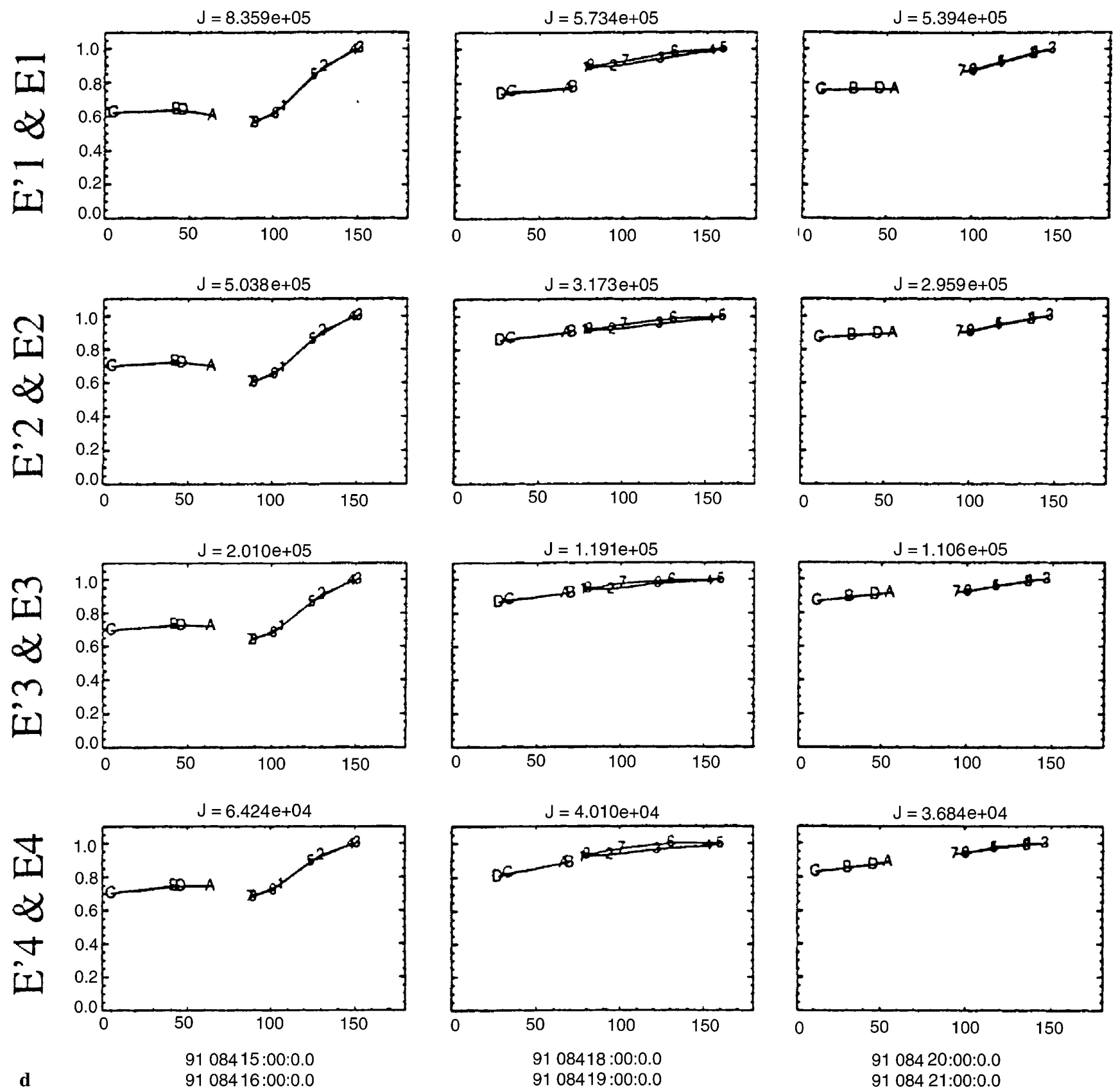

Fig. 2d. The electron population detected in region $D$ exhibits unidirectional anisotropies directed anti-parallel to the IMF direction. Apparently electrons are streaming away from the Sun during this period.

$\sim \mathrm{W} 60^{\circ}$ on the Sun as viewed from the Earth. The delayed onset and slow rise time (e-folding time equal to $3.1 \mathrm{~h}$ ) of the electron event is to be expected from the consistently "poor" nominal magnetic connection with the flare longitude. Furthermore, the slow rise time of the electron event studied and the non-impulsive nature of its rise phase indicate that the particles detected did not have direct access to the spacecraft after their injection at the Sun. In contrast, initially they must have undergone drifts across the highly inhomogeneous magnetic fields prevailing near the Sun, before reaching the Ulysses footpoint and escaping in the interplanetary medium along the magnetic field lines.

We emphasize the fact that the electrons observed were not accelerated by the CME-driven shocks. According to Cane et al. (1988), an abrupt decrease in the particle fluxes is expected to be observed as the spacecraft enters the CME. However, on the contrary, in 
REGION “E”
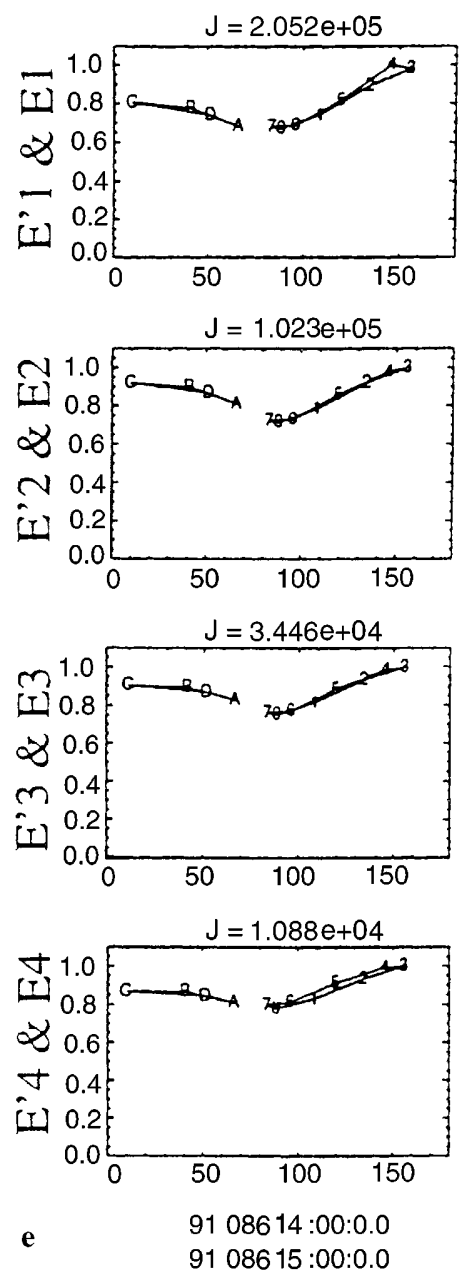

\section{REGION "F"}
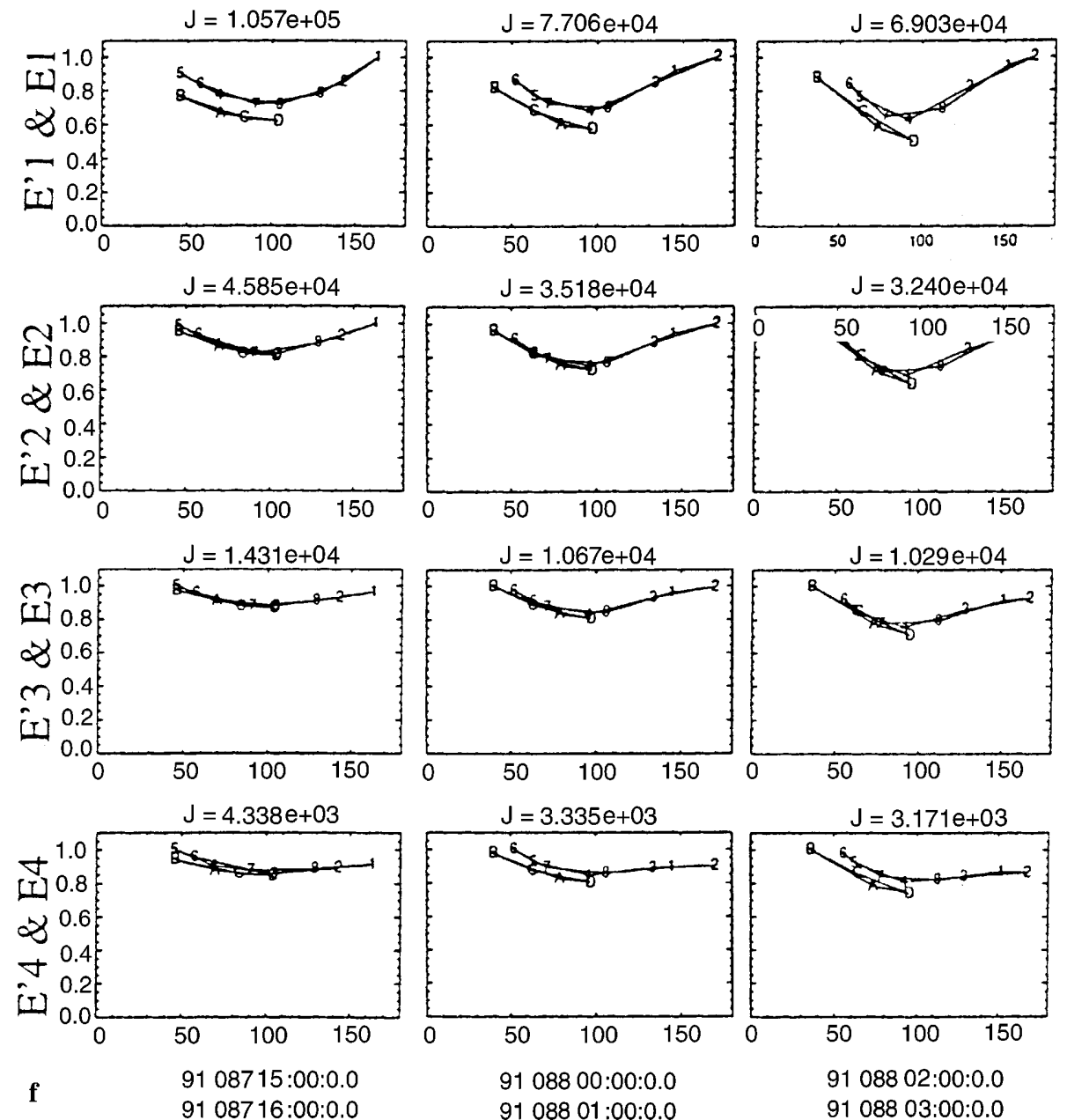

Fig. 2e,f. e The electrons in region $E$ also show a trapping distribution for $\sim 3$ h within the second ICMEF. f Representative electron PAD plots for region $F$ where strong and persistent bi-directionalities indicate the trapping of these particles.

March 1991, an enhancement is observed at the onset of the first CME and no variation in the particle flux is observed for the second CME.

After the shock disturbance has passed by, the electron fluxes begin a smooth, slow, quasi-exponential decay. Such a long and smooth decay to ambient values is typically observed during the decay phase of impulsive solar flare electron events (Lin, 1974). On the basis of these particle flux profile features, it is concluded that the particle flux variations observed during the passage of the two ICMEs are clearly temporal. This deduction is further corroborated by the fact that the smooth decay phase of the event observed is not interrupted by any modulations of the electron intensity, apparently convected by the solar wind. On the contrary, Roelof et al. (1992) have observed convective modulations during the rise phase of this event. Moreover, based upon the particle flux profile characteristics, we argue that the magnetic flux tubes that populated the interior region of the ICMEs with energetic particles throughout this period connected back to the same particle source region on the Sun. Also, the fact that the ICMEs observed are obviously transparent to SEPs suggests that we are not dealing with closed field structures detached from the Sun. Assuming SEPs are injected into interplanetary space from the Sun while a closed magnetically isolated field structure or plasmoid is present in space, those particles should be excluded from the region of the plasmoid, as they would have to propagate mainly across magnetic field lines to enter it. The passage of such regions past Ulysses should therefore be accompanied by substantial reductions in SEP fluxes, contrary to what is observed. Thus, it can be concluded that the magnetic field structures embedded within the ICMEs convected over Ulysses at the end of March 1991, were still anchored at the Sun when the ICMEs arrived at 2.5 AU.

The unusual bi-directional electron anisotropies observed during the passage of the two ICMEs over Ulysses can be interpreted as evidence for the existence of trapped electron populations within portions of the ICME structures. A magnetic field configuration allow- 


\section{REGION “G”}
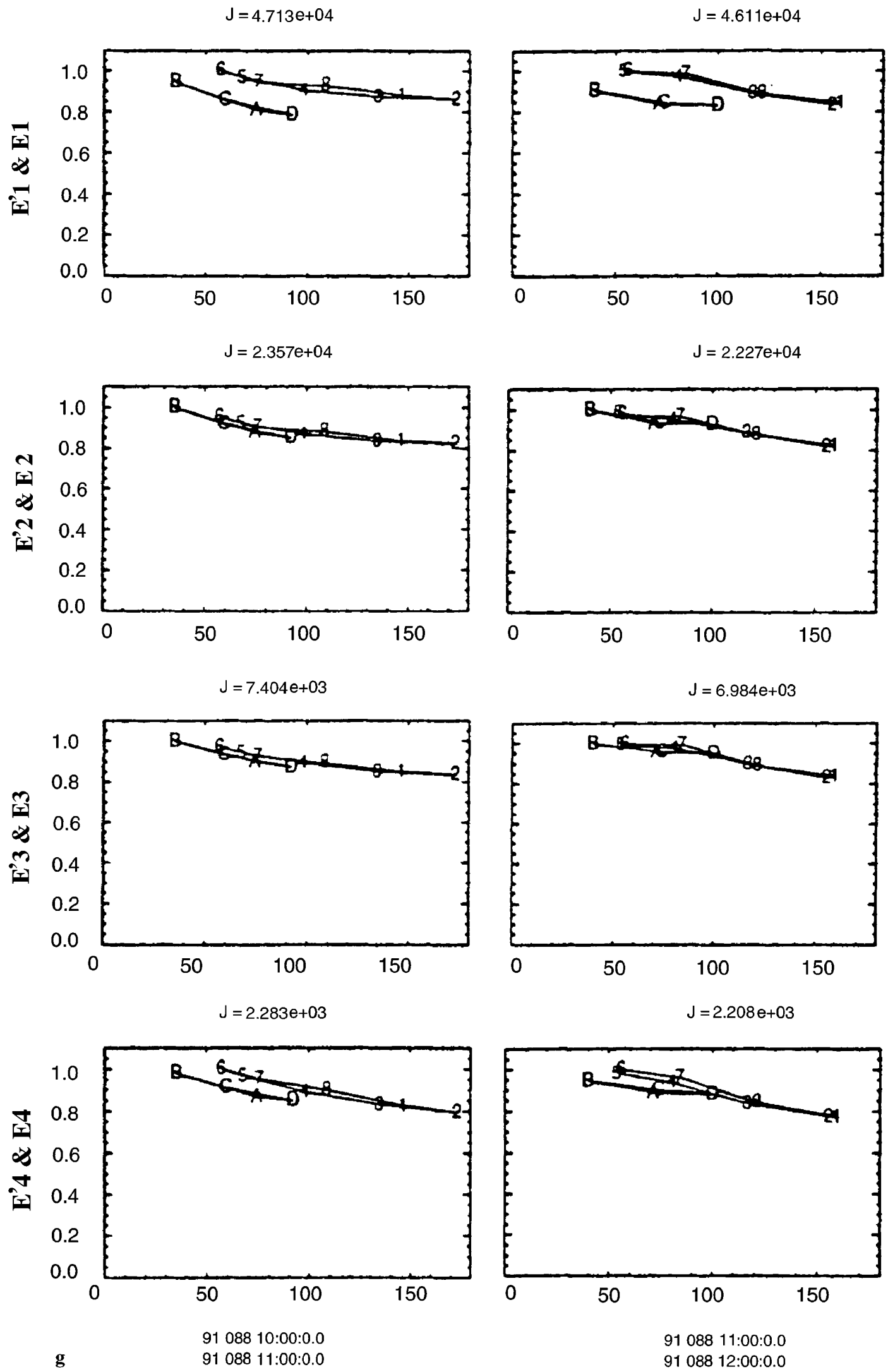

Fig. 2g Three hours before exiting the rear boundary of the second ICME Ulysees detects region $G$, where electrons are streaming away from the Sun 
ing trapping of energetic particles is the existence of magnetic loops connected to the solar corona at both ends (Gosling, 1990). The energetic particles following their injection at the Sun are trapped into the magnetic "loop-like" structure and bounce back and forth between two magnetic mirrors which are located near the Sun. Moreover, another candidate magnetic field topology that can effectively trap particles is an open magnetic field configuration. In this case, one magnetic mirror is located near the Sun and a magnetic constriction beyond the spacecraft, formed by the convergence of open field lines which reflects the outgoing electrons and creates the observed bi-directional electron anisotropies (Sarris et al., 1983; Malandraki et al., 1997).

Our observations of counterstreaming $42-290 \mathrm{keV}$ electrons are consistent with the trapping of particles in closed magnetic loop-like structures embedded within portions of the ICMEs convected over Ulysses. Thus, within regions $\mathrm{A}$ and $\mathrm{B}$, Ulysses detects bi-directional electron anisotropies produced by particles, initially injected by solar flares into magnetic loop-like structures anchored at the Sun and extending beyond the shock front (Fig. 4). The solar flare particles are trapped within the magnetic loops attached to the Sun, bouncing back and forth between mirrors located at the Sun. These strong trapping signatures, namely the bi-directional anisotropies, were observed both before and after the shock wave. Furthermore, we likely suggest that the strong bi-directional electron anisotropies observed in region $\mathrm{C}$ can be explained by the trapping configuration of a closed loop-like structure anchored at the Sun at both ends. The configuration of a magnetic constriction formed beyond the spacecraft where particles are backscattered cannot be definitely ruled out for these regions since mapping through extrapolation of the magnetic field and solar wind in the vicinity of shocks and CIRs is inaccurate. It is considered to be less probable for region $\mathrm{C}$, where the counterstreaming electron population is detected, because it is a region with the highest magnetic field magnitude in the whole period, in contrast to what is anticipated for trapping regions in between magnetic mirrors (Vandas et al., 1996). Finally, the unidirectional electron anisotropies detected in region $\mathrm{D}$ provide evidence that the IMF structures encountered by Ulysses may be magnetically open, namely connected to the Sun at one end (Fig. 4).

Using a similar line of thought to analyze the anisotropy regimes identified within the second ICME, we consider that the observations of counterstreaming electrons in region $\mathrm{E}$ are consistent with the presence of a closed magnetic field loop-like structure connected at both ends at the Sun. No magnetic constriction can be identified beyond the spacecraft to account for the observed bi-directional anisotropies since the solar wind speed (courtesy NSSDC, not shown) is smoothly declining after the passage of region $\mathrm{E}$ over Ulysses. However, since the solar wind speed increases abruptly after the passage of region $\mathrm{F}$, counterstreaming electrons observed in this region are possibly the result of mirroring of the electrons on a downstream magnetic mirror region arising from colliding solar wind streams
(Anderson et al., 1995). Magnetically open IMF lines only, rooted at one end in the solar corona, can apparently account for the streaming electrons away from the Sun, observed while Ulysses is traversing region $\mathrm{G}$.

In Fig. 3, the absolute magnitude of the $65-112 \mathrm{keV}$ electron anisotropy $\left(j_{\max } / j_{\min }\right)$ has been plotted with time, for the $52 \mathrm{~h}$ period from entrance of Ulysses in region A till exit from the first ICME. Squares denote the bi-directional anisotropy value for each hourlyaveraged measurement. Crosses indicate periods where unidirectional anisotropies were observed. As can be seen in Fig. 3, bi-directional and unidirectional anisotropies cluster around specific anisotropy values within the ICME. This may indicate Ulysses detects magnetic field filaments within which bi-directional electron anisotropies obtain distinct values (filaments with distinct bi-directional electron anisotropy characteristics). IMF filaments with unidirectional particle intensities are also detected as well as flux tubes with isotropic distributions. Thus, magnetic field regimes with different flux tube configurations and anisotropy characteristics embedded within the ICME are observed. Bi-directional anisotropies indicate the crossing of magnetic loops extending beyond $2.5 \mathrm{AU}$, whereas streaming electrons away from the Sun suggests that the IMF structure encountered is magnetically open, thus allowing the escape of particles to deep space. As is clear in Fig. 3, the two curves drawn form envelopes within which all the values of the electron bi-directional anisotropies for two distinct filaments are confined. Evidently, the amplitude of the bi-directional anisotropy in the filaments embedded within the first ICME decreases steeply with time.

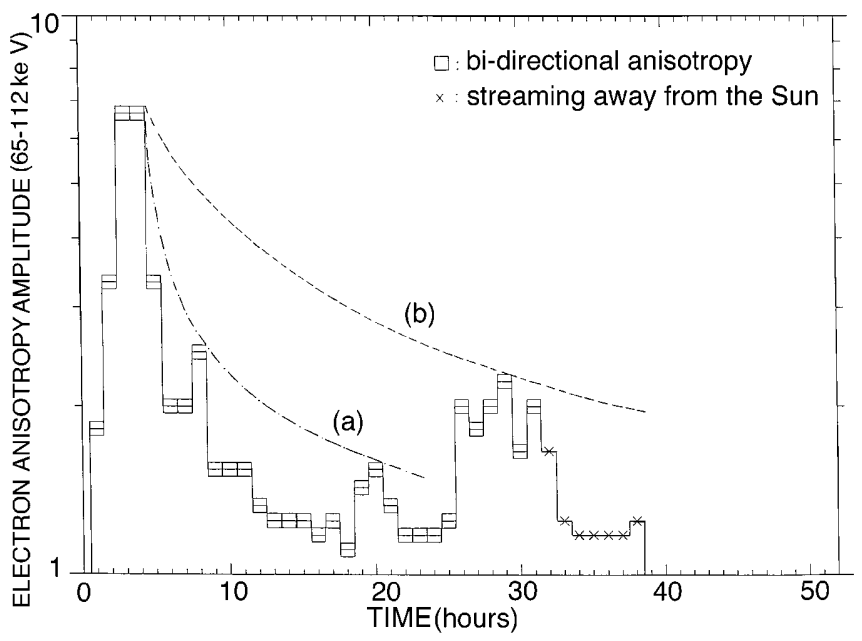

Fig. 3. The absolute magnitude of the $65-112 \mathrm{keV}$ electron anisotropy $\left(j_{\max } / j_{\text {min }}\right)$ is plotted versus time for the $52 \mathrm{~h}$ period from entrance of Ulysses in region $A$ till exit from the first ICME. Squares indicate the bi-directional anisotropy value for each hourly-averaged measurement, whereas crosses denote unidirectional anisotropies. Curves $a$ and $b$ form envelopes within which all the values of the electron bi-directional anisotropies for two distinct filaments are confined 
We have selected magnetic flux tubes embedded within the first ICME with bi-directional electron pitch angle distributions which are indicative of closed field structures and we follow the decay of the peak values of the bidirectional anisotropy as Ulysses receeds from the onset of the ICME. Assuming an exponential decay for the anisotropy amplitude of the electron population trapped in the various filaments embedded within the ICME:

$A(t)=A_{0} \exp (-t / \tau)$

the anisotropy decay time constants $\tau_{1}$ and $\tau_{2}$ were computed for the two curves (a) and (b) in Fig. 3 and found to be 9 and $22 \mathrm{~h}$ respectively, indicating a lower and an upper limit correspondingly. The chosen bidirectional anisotropy values from the envelopes (Fig. 3) correspond to approximately the same omnidirectional particle intensity values (Fig. 1). However, the anisotropy amplitudes of the particle intensities decrease. This is a measure of the evolution of the anisotropy which in turn is a measure of the scattering of the electrons trapped within the ICME loop structures.

In Fig. 4, a representative schematic is shown of possible open and closed configuration of the IMF, embedded within the first ICME (regions A, B, C, and D). Ulysses proceeds along the dashed line, shown in Fig. 4, and is thus probing magnetic field regimes with different flux tube configurations.

\section{Conclusions}

In this work, we present an examination of the time evolution of the angular distributions of the solar flare electron fluxes ( $E \geq 42 \mathrm{keV})$ observed during the passage of two ICMEs over Ulysses, near the end of March 1991. The investigation revealed several distinct extended magnetic flux tubes corresponding to different electron regimes, adjacent to each other in some cases, that were associated with distinctly different and unusual anisot-

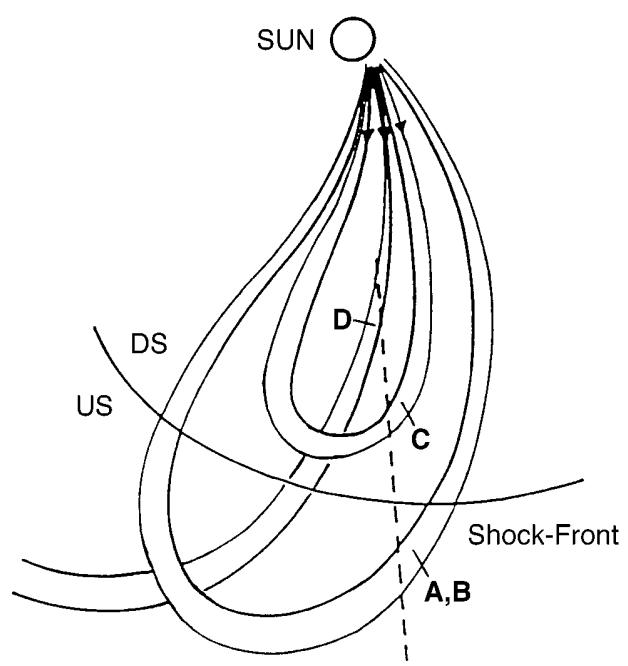

Fig. 4. Sketch of a possible closed (regions $A, B, C$ ) and open (region $D$ ) IMF configuration embedded within the first ICME as evidenced by observations of energetic electron anisotropies. The dashed trace marks Ulysses trajectory ropy characteristics. Particle observations have provided significant information on the internal structure of the ICMEs. Portions of the first ICME apparently had the structure of a magnetic cloud consisting of closed magnetic field lines connected at both ends at the Sun, as supported by counterstreaming electrons along the local IMF. The configuration of a magnetic mirror region formed beyond the spacecraft cannot be definitely excluded for this ICME. Other portions of the ICME detected in the downstream region of the shock most likely consisted of open magnetic filaments connected to the Sun at only one end and allowing escape of particles to deep space. This interpretation is supported by the observation of unidirectional electron anisotropies (region $\mathrm{D}$ ), persisting for $\sim 7 \mathrm{~h}$, which indicate that electrons are streaming away from the Sun.

The field-aligned bi-directional electron anisotropies observed within region $\mathrm{E}$ of the second ICME are consistent with the existence of closed loop-like structures rooted at both ends in the solar corona. The convergence of open field lines forming a magnetic constriction beyond Ulysses can possibly explain the observed counterstreaming electrons within region $\mathrm{F}$. The trailing portion of this ICME obviously consists of open field lines rooted at only one end in the solar corona, as supported by the observed electrons streaming away from the Sun.

We conclude from these findings that the topologies of some CMEs are more complex than expected, involving open and closed magnetic field topologies. We find support for the findings of Gosling et al. (1995) and Bothmer et al. (1996). According to Gosling et al. (1995), such magnetic field topologies are likely created by sustained 3-dimensional magnetic reconnection of coronal loops in the rising phase of CMEs.

Furthermore, a measure of the scattering of the electrons trapped within the looped structures of the first ICME was obtained, which shows that for the two distinct filaments [curves (a), (b) in Fig. 3] the electron anisotropy peak values have decreased down to $\sim 1 / 3$ of their initial value in 9 and $22 \mathrm{~h}$ respectively. The anisotropy decrease is a result of the encounter of magnetic irregularities by the relativistic electrons which are trapped along the ICME loop-like structures.

In conclusion, the presented particle observations show that the magnetic field structures embedded within the ICMEs studied are rooted at the Sun allowing direct access of solar flare electrons, which argues against a detached loop or plasmoid topology for these ICMEs.

Acknowledgements. We are thankful to Mr. Nick Sidiropoulos for his assistance with data processing and to the National Observatory of Athens for the hospitality extended to one of us (OM) and use of its facilities during part of this work.

Topical Editor R. Schwenn thanks V. Bothmer and M. Pick for their help in evaluating this paper.

\section{References}

Anderson, K. A., J. Sommers, R. P. Lin, M. Pick, P. Chaizy, N. Murphy, E. J. Smith, and J. L. Phillips, Mirroring of fast 
solar electrons on a downstream corotating interaction region, J. Geophys. Res., 100, 3, 1995.

Armstrong, T. P., D. Haggerty, L. J. Lanzerotti, C. G. Maclennan, E. C. Roelof, M. Pick, G. M. Simnett, R. E. Gold, S. M. Krimigis, K. A. Anderson, R. P. Lin, E. T. Sarris, R. Forsyth, and A. Balogh, Observations by Ulysses of hot $(\sim 270 \mathrm{keV})$ coronal particles at $32^{\circ}$ south heliolatitude and 4.6 AU, Geophys. Res. Lett., 21, 1747, 1994.

Balogh, A., T. J. Beek, R. J. Forsyth, P. C. Hedgecock, R. J. Marquedant, E. J. Smith, D. J. Southwood, and B. T. Tsurutani, The magnetic field investigation on the Ulysses mission: instrumentation and preliminary scientific results, Astron. Astrophys. Suppl. Ser., 92, 207, 1992.

Balogh, A., J. A. Gonzalez-Esparza, R. J. Forsyth, M. E. Burton, B. E. Goldstein, E. J. Smith, and S. J. Bame, Interplanetary shock waves: Ulysses observations in and out of the ecliptic plane, Space Sci. Rev., 73, H171, 1995.

Bothmer, V., R. G. Marsden, T. R. Sanderson, K. J. Trattner, K.-P. Wenzel, A. Balogh, R. J. Forsyth, and B. E. Goldstein, The Ulysses south polar pass: transient fluxes of energetic ions, Geophys. Res. Lett., 22, 3369, 1995.

Bothmer, V., M. I. Desai, R. G. Marsden, T. R. Sanderson, K. J. Trattner, K.-P. Wenzel, J. T. Gosling, A. Balogh, R. J. Forsyth, and B. E. Goldstein, Ulysses observations of open and closed magnetic field lines within a coronal mass ejection, Astron. Astrophys., 316, 493, 1996.

Burlaga, L. F., Micro-scale structures in the interplanetary medium, Sol. Phys., 4, 67, 1968.

Cane, H. V., D. V. Reames, and T. T. von Rosenvinge, The role of interplanetary shocks in the longitude distribution of solar energetic particles, J. Geophys. Res., 93, 9555, 1988.

Cocconi, G., K. Greisen, P. Morrison, T. Gold, and S. Hayakawa, The cosmic ray flare effect, Nuovo Cimento, Suppl., 8, 161, 1958.

Farrugia, C. J., L. F. Burlaga, V. A. Osherovich, I. G. Richardson, M. P. Freeman, R. P. Lepping, and A. J. Lazarus, A study of an expanding interplanetary magnetic cloud and its interaction with the Earth's magnetosphere: the interplanetary aspect, J. Geophys. Res., 98, 7621, 1993.

Fischer, S., M. Vandas, A. Geranios, P. Trochoutsos, and E. Sarris, Bidirectional particle fluxes in magnetic clouds and their environs, in Proc. $25^{\text {th }}$ Int. Cosmic Ray Conf., Durban, South Africa, 1, 349, 1997.

Gold, T., Plasma and magnetic fields in the solar system, J. Geophys. Res., 64, 1665, 1959.

Gosling, J. T., Coronal mass ejections and magnetic flux ropes in interplanetary space, in Physics of Magnetic Flux Ropes, Geophys. Monogr. Ser., vol 58, Eds. C. T. Russell, E. R. Priest, and L. C. Lee, p. 343, AGU, Washington, D. C., 1990.

Gosling, J. T., E. Hildner, R. M. MacQueen, R. H. Munro, A. I. Poland, and C. L. Ross, Mass ejections from the Sun: a view from Skylab, J. Geophys. Res., 79, 4581, 1974.

Gosling, J. T., J. Birn, and M. Hesse, Three-dimensional magnetic reconnection and the magnetic topology of coronal mass ejection events, Geophys. Res. Lett., 22, 869, 1995.

Kahler, S. W., and D. V. Reames, Probing the magnetic topologies of magnetic clouds by means of solar energetic particles, J. Geophys. Res., 96, 9419, 1991.

Kutchko, F. J., R. P. Briggs, and T. P. Armstrong, The bidirectional particle event of October 12, 1977, possibly associated with a magnetic loop, J. Geophys. Res., 87, 1419, 1982.

Lanzerotti, L. J., R. E. Gold, K. A. Anderson, T. P. Armstrong, R. P. Lin, S. M. Krimigis, M. Pick, E. C. Roelof, E. T. Sarris, G. M. Simnett, and W. E. Frain, Heliosphere instrument for spectra, composition and anisotropy at low energies, Astron. Astrophys. Suppl. Ser., 92, 349, 1992.

Larson, D. E., R. P. Lin, J. M. McTiernan, J. P. McFadden, R. E. Ergun, M. McCarthy, H. Reme, T. R. Sanderson, M. Kaiser, R. P. Lepping, and J. Mazur, Tracing the topology of the October 18-20, 1995, magnetic cloud with $\sim 0.1-10^{2} \mathrm{keV}$ electrons, Geophys. Res. Lett., 24, 1911, 1997.

Lin, R. P., Non-relativistic solar electrons, Space Sci. Rev., 16, 189, 1974.

Maia, D., O. Malandraki, M. Pick, E. T. Sarris, G. Kasotakis, L. J. Lanzerotti, C. G. MacLennan, and P. C. Trochoutsos, Particle propagation channel detected at 4.7 AU inside a corotating interaction region, J. Geophys. Res., 103, 9545, 1998.

Malandraki O., Gr. Kasotakis, E. T. Sarris, P. Trochoutsos, D. Dialetis, and G. Tsiropoula, Solar flare electrons propagation in converging interplanetary magnetic structures, in: Proc. $25^{\text {th }}$ Int. Cosmic Ray Conf., Durban, South Africa, 1, 281, 1997.

Malandraki, O., E. T. Sarris, Gr. Kasotakis, and N. Sidiropoulos, Study of CME structure and evolution deduced from ULYSSES/HI-SCALE energetic particle observations, Adv. Space. Res., in press, 2000.

Marsden, R. G., T. R. Sanderson, C. Tranquille, K.-P. Wenzel, and E. J. Smith, ISEE 3 Observations of low-energy proton bidirectional events and their relation to isolated interplanetary magnetic structures, J. Geophys. Res., 92, $11009,1987$.

Palmer, I. D., F. R. Allum, and S. Singer, Bidirectional anisotropies in solar cosmic ray events: evidence for magnetic bottles, J. Geophys. Res., 83, 75, 1978.

Phillips, J. L., Coronal mass ejections encountered by the Ulysses spacecraft during the in-ecliptic mission phase, Los Alamos Report, LA-UR, 1087, 1997.

Phillips, J. L., S. J. Bame, J. T. Gosling, D. J. McComas, B. E. Goldstein, E. J. Smith, A. Balogh, and R. J. Forsyth, Ulysses plasma observations of coronal mass ejections near $2.5 \mathrm{AU}$, Geophys. Res. Lett., 19, 1239, 1992.

Pick M., L. J. Lanzerotti, A. Buttighoffer, E. T. Sarris, T. P. Armstrong, G. M. Simnett, E. C. Roelof, and A. Kerdraon, The propagation of sub-MeV solar electrons to heliolatitudes above 50S, Geophys. Res. Lett., 22, 3373, 1995.

Piddington. J. H., Interplanetary magnetic field and its control of cosmic-ray variations, Phys. Res., 119, 589, 1958.

Rao U. R., K. G. McCracken, and R. P. Bukata, Cosmic ray propagation processes, 2, the energetic storm particle event, J. Geophys. Res., 72, 4325, 1967.

Richardson I.G., Using energetic particles to probe the magnetic topology of ejecta, in Coronal Mass Ejections, Geophys. Monogr. Ser., vol 99, Eds. N. Crooker, J. A. Joselyn, J. Feynman, p. 189, AGU, Washington, 1997.

Roelof E. C., R. E. Gold, G. M. Simnett, S. J. Tapping, T. P. Armstrong, and L. J. Lanzerotti, Low-energy solar electrons and ions observed at Ulysses February-April, 1991: the inner heliosphere as a particle reservoir, Geophys. Res. Lett., 19, 1243, 1992.

Sarris E. T., and S. M. Krimigis, Evidence for solar magnetic loops beyond 1 AU, Geophys. Res. Lett., 9, 167, 1982.

Sarris E. T., P. C. Trochoutsos, and G. C. Anagnostopoulos, Study on the onsets of solar energetic electron events, Sol. Phys., 83, 51,1983

Vandas M., S. Fischer, A. Geranios, M. Dryer, Z. Smith, and T. Detman, Magnetic traps in the interplanetary medium associated with magnetic clouds, J. Geophys. Res., 101, $21589,1996$. 